\title{
Solubility of butylated hydroxytoluene (BHT) in aqueous and alcohol solutions from 293.15 to $313.15 \mathrm{~K}$
}

\author{
Shipra Baluja ${ }^{1, \star}$, Kapil Bhesaniya ${ }^{1}$, Rahul Bhalodia $^{1}$, Sumitra Chanda ${ }^{2}$ \\ ${ }^{1}$ Physical Chemical Laboratory, Department of Chemistry, Saurashtra University, Rajkot, India \\ ${ }^{2}$ Bio-Science Department, Saurashtra University, Rajkot - 360 005, India \\ *E-mail address: shipra_baluja@rediffmail.com
}

\begin{abstract}
The solubility of Butylated hydroxyl toluene in aqueous and alcohol solutions were determined at different temperatures (293.15 to 313.15) K. Using Van't Hoff and Gibb's equations, some thermodynamic functions such as Gibbs energy, enthalpy and entropy of dissolution, and of mixing of Butylated hydroxyl toluene in aqueous and alcohol solutions, were evaluated from solubility data. The solubility was greater in butanol and minimum in water. The enthalpies, entropy and Gibb's energy of dissolution were positive for all solvents.
\end{abstract}

Keywords: Butylated hydroxytoluene (BHT); Average deviation; Relative deviation; Gibbs energy

\section{INTRODUCTION}

Butylated hydroxytoluene (BHT) [IUPAC: 2,6-di-tert-butyl-4-methylphenol] (Fig. 1) is a lipophilic phenol, primarily used as an antioxidant [1-2] food additive as well as in cosmetics, pharmaceuticals, jet fuels, rubber, petroleum products, and embalming fluid [3-5]. It behaves as a synthetic analogue of vitamin E [6], primarily acting as a terminating agent that suppressed auto oxidation.

These applications prompted us to study the solubility of BHT in different solvents such as methanol, ethanol, propanol, butanol and water. The study is done at different temperatures (293.15 to $313.15 \mathrm{~K})$.

The data may be useful for the design process of pharmaceutical dosage form. Further, the study of temperature dependence solubility data provides the explanation of molecular mechanisms involved in the respective drug dissolution process.

\section{EXPERIMENTAL}

\section{1. Materials}

BHT, a white powder with a purity of 99.6 mass \%, was purchased from Himedia Pvt. Ltd. All the solvents, methanol, ethanol, propanol, butanol and water were analytical grade 
reagents and were purified by fractional distillation. Their purities were checked by SHIMADZU GC-MS (Model No QP-2010) and were found to be greater than $99.85 \%$. Melting point of BHT was determined by DSC and was found to be in agreement with the reported value [7].

\section{2. Equipments}

Mettler Toledo AB204-S, electronic balance was used with an accuracy of $\pm 0.1 \mathrm{mg}$. The UV spectrophotometer of Shimadzu make was used for concentration determination.

\section{3. Solubility measurement}

An excess mass of BHT was added to a known mass of solvent in stoppered glass flasks. The solid-liquid mixtures were placed on an ultrasonic bath for about 15 minutes and were stirred in a mechanical shaker for one hour. The samples were then allowed to stand in water bath kept at appropriate temperature with $\pm 0.05 \mathrm{~K}$. All samples were maintained at least for 48 hours to reach the equilibrium. This equilibrium time was established by quantifying the BHT concentration to obtain a constant value. After this time, the supernatant solutions were filtered (at isothermal conditions) to insure ensure that they are free of particulate matter before sampling. The concentrations were determined by measuring the absorbance after appropriate dilution and interpolation from previously constructed UV-spectrophotometric calibration curves. All the measurements were repeated at least three times.

\section{RESULTS AND DISCUSSIONS}

Tables 1 and 2 summarize the physicochemical properties of Butylated hydroxytoluene (BHT) and of studied solvents respectively.

Table 1. Physical Data for the selected solvents.

\begin{tabular}{|c|c|c|c|c|}
\hline Solvent & $\begin{array}{c}\text { Molar mass } \\
\left(\mathrm{g} \cdot \mathrm{mol}^{-1}\right)\end{array}$ & $\begin{array}{c}\text { Boiling point } \\
\left({ }^{\circ} \mathrm{C}\right)\end{array}$ & $\begin{array}{c}\text { Dielectric } \\
\text { constant }\end{array}$ & $\begin{array}{c}\text { Dipole } \\
\text { moment }\end{array}$ \\
\hline Water & 18 & 100 & 80 & - \\
\hline Methanol & 32 & 64.6 & 33 & 1.70 \\
\hline Ethanol & 46 & 79 & 24.55 & $1.69 \mathrm{D}$ \\
\hline Propanol & 60 & 97 & 20 & $1.68 \mathrm{D}$ \\
\hline Butanol & 74 & 117.6 & 18 & $1.63 \mathrm{D}$ \\
\hline
\end{tabular}


Table 2. Physicochemical Data of BHT.

\begin{tabular}{|c|c|c|c|}
\hline $\begin{array}{c}\text { Molar mass } \\
\left(\mathrm{g} \cdot \mathrm{mol}^{-1}\right)\end{array}$ & $\begin{array}{c}\text { Melting } \\
\text { point }(\mathrm{K})\end{array}$ & $\begin{array}{c}\Delta H_{\text {fus }} \\
\left(\mathrm{kJ} \cdot \mathrm{mol}^{-1}\right)\end{array}$ & $\begin{array}{c}\Delta S_{f u s} \\
\left(\mathrm{~J} \cdot \mathrm{mol}^{-1}\right)\end{array}$ \\
\hline 220.35 & 344.15 & 19.87 & 57.7365 \\
\hline
\end{tabular}<smiles>Cc1cc(C(C)(C)C)c(O)c(C(C)(C)C)c1</smiles>

\section{2,6-di-tert-butyl-4-methylphenol}

Figure 1. Molecular structure of BHT.

\section{1. Ideal and experimental solubility of BHT}

The ideal solubility of a crystalline solute in a liquid solvent can be calculated by the following equation:

$$
\ln x_{2}^{i d}=-\frac{\Delta H_{\text {fus }}\left(T_{\text {fus }}-T\right)}{R T_{\text {fus }} T}+\left(\frac{\Delta C_{P}}{R}\right)\left[\frac{\left(T_{f u s}-T\right)}{T}+\ln \left(\frac{T}{T_{\text {fus }}}\right)\right]
$$

where $\mathrm{x}_{2}{ }^{\text {id }}$ is the ideal solubility of solute as mole fraction, $\Delta H_{f u s}$ is the molar enthalpy of fusion of the pure solute, $T_{\text {fus }}$ is the absolute melting point, $\mathrm{T}$ is the absolute solution temperature, $\mathrm{R}$ is the gas constant $\left(8.314 \mathrm{~J} \mathrm{~mol}^{-1} \mathrm{~K}^{-1}\right)$ and $\Delta C_{p}$ is the difference between the molar heat capacity of the crystalline form and the molar heat capacity of the hypothetical super cooled liquid form, both at solution temperature [8]. $\Delta C_{p}$ can't be easily determined so it is assumed that $\Delta C_{p}$ is approximately equal to entropy of fusion $\Delta S_{f u s}$ [9].

The ideal solubilities of BHT at different temperatures are given in Table 3 whereas experimental solubilities of BHT in water and alcohol solutions at different temperatures ( 293.15 to $313.15 \mathrm{~K}$ ) are summarized in Table 4 . The variation of solubility with temperature is also shown in Figure 2. It is observed that solubility increases linearly with increase in temperature. Further, solubility is higher in butanol and minimum in water. This suggests that solubility increases with increase in $-\mathrm{CH}_{2}$ group. Further, the minimum solubility of water suggests that BHT structure leads to water association which is not favorable for solubility of BHT in water whereas butanol could form maximum hydrogen bonding with BHT which causes increase in solubility. 
Table 3. Ideal Solubilities $\left(x_{2}^{i d}\right)$ of BHT at different temperatures.

\begin{tabular}{|c|c|c|c|c|c|c|c|c|}
\hline Temp. (K) & 293.15 & 295.15 & 298.15 & 303.15 & 305.15 & 208.17 & 210.15 & 313.15 \\
\hline$x_{2}{ }^{\text {id }}$ & 0.3283 & 0.3441 & 0.3692 & 0.3868 & 0.4144 & 0.4338 & 0.4856 & 0.5192 \\
\hline
\end{tabular}

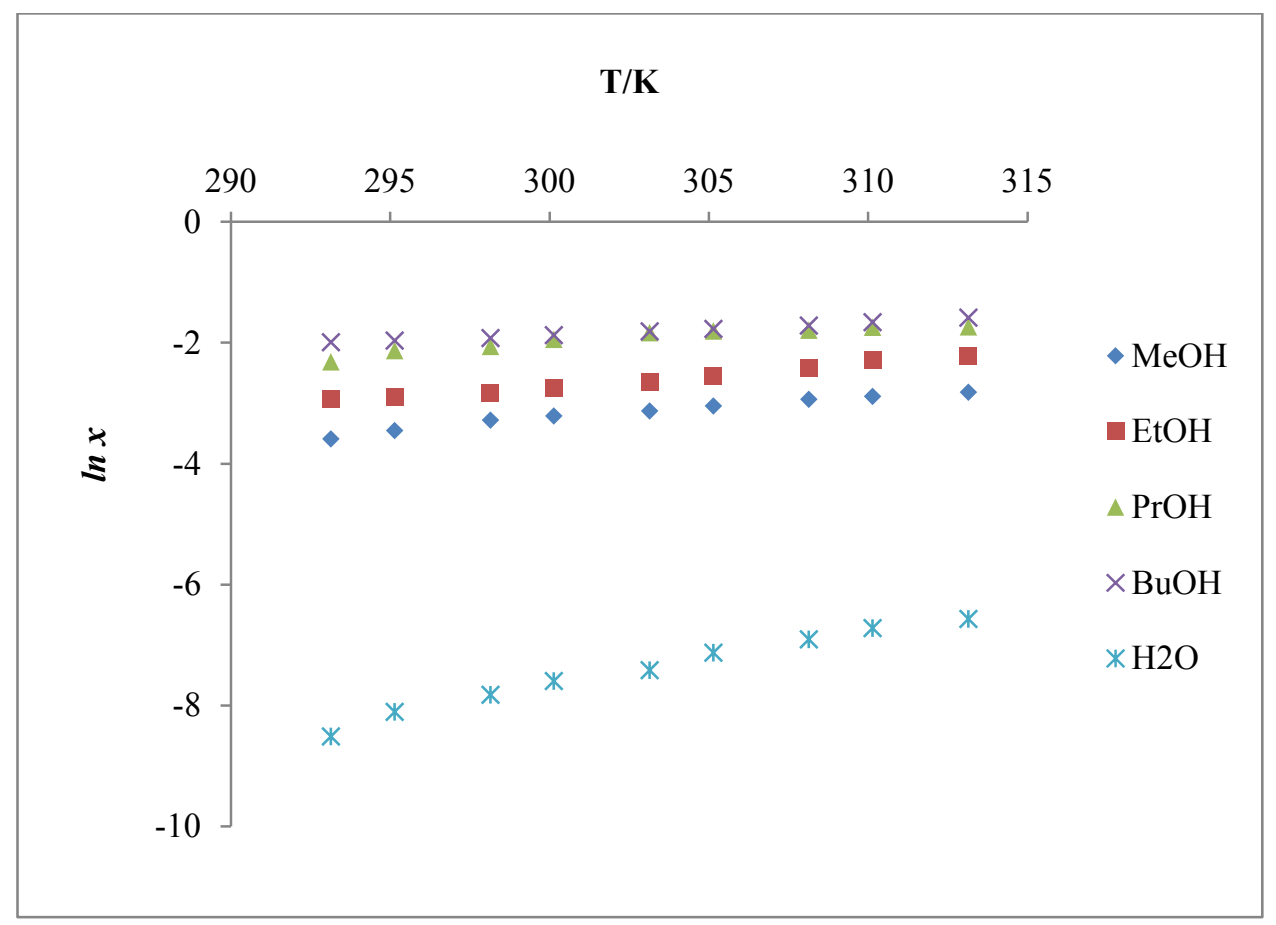

Figure 2. Solubility $x$ of BHT as a function of temperature.

The mole fraction solubility $x$ of BHT was also correlated as a function of temperature by the modified Apelblat equation [10-11]

$$
\ln x=A+B(T / K)
$$

where $x$ is the mass fraction solubility of BHT; $T$ is the absolute temperature and $A$ and $B$ are the coefficients in equation (2). The values of these coefficients are given in Table 5. The calculated solubilities $x_{c i}$ are also reported in Table 4. Further, absolute average deviations $(A A D)$ and root-mean-square deviations $(r m s d)$, calculated by equations (3) and (4) are listed in Table 5.

$$
A A D=\frac{1}{N} \sum_{i}^{N} \frac{x_{i}-x_{c i}}{x_{i}}
$$




$$
r m s d=\left[\sum_{i=1}^{N} \frac{\left(x_{c i}-x_{i}\right)^{2}}{N-1}\right]^{1 / 2}
$$

Table 4. Experimental Mole Fraction Solubilities $(x)$, Calculated Mole Fraction Solubilities $\left(x_{c i}\right)$ and relative deviation $(R D)$ of $\mathrm{BHT}$ in different solvents.

\begin{tabular}{|c|c|c|c|c|c|c|}
\hline$T / \mathrm{K}$ & $10^{-2} x$ & $10^{-2} x_{c i}$ & $\begin{array}{l}100 \\
\text { RD }\end{array}$ & $10^{-2} x$ & $10^{-2} x_{c i}$ & $\begin{array}{l}100 \\
\text { RD } \\
\end{array}$ \\
\hline \multicolumn{4}{|c|}{ Methanol } & \multicolumn{3}{|c|}{ Ethanol } \\
\hline 293.15 & $2.75 \pm 0.02$ & $3.18 \pm 0.01$ & -0.36 & $5.31 \pm 0.03$ & $5.06 \pm 0.02$ & -1.60 \\
\hline 295.15 & $3.16 \pm 0.02$ & $3.40 \pm 0.01$ & 2.12 & $5.52 \pm 0.02$ & $5.47 \pm 0.01$ & -0.36 \\
\hline 298.15 & $3.76 \pm 0.01$ & $3.76 \pm 0.01$ & -0.02 & $5.83 \pm 0.01$ & $6.13 \pm 0.01$ & 1.75 \\
\hline 300.15 & $4.01 \pm 0.03$ & $4.02 \pm 0.02$ & 0.06 & $6.32 \pm 0.04$ & $6.62 \pm 0.02$ & 1.64 \\
\hline 303.15 & $4.37 \pm 0.01$ & $4.44 \pm 0.01$ & 0.49 & $7.05 \pm 0.05$ & $7.43 \pm 0.03$ & 1.93 \\
\hline 305.15 & $4.75 \pm 0.02$ & $4.75 \pm 0.02$ & 0.02 & $7.77 \pm 0.06$ & $8.02 \pm 0.02$ & 1.22 \\
\hline 308.15 & $5.30 \pm 0.04$ & $5.26 \pm 0.02$ & -0.31 & $8.83 \pm 0.02$ & $8.99 \pm 0.03$ & 0.75 \\
\hline 310.15 & $5.57 \pm 0.03$ & $5.62 \pm 0.01$ & 0.31 & $10.10 \pm 0.03$ & $9.71 \pm 0.01$ & -1.75 \\
\hline 313.15 & $5.95 \pm 0.04$ & $6.22 \pm 0.03$ & 1.54 & $10.95 \pm 0.06$ & $10.89 \pm 0.02$ & -0.23 \\
\hline \multicolumn{4}{|c|}{ Propanol } & \multicolumn{3}{|c|}{ Butanol } \\
\hline 293.15 & $9.85 \pm 0.05$ & $10.98 \pm 0.03$ & 4.70 & $13.58 \pm 0.03$ & $13.42 \pm 0.02$ & -0.62 \\
\hline 295.15 & $11.87 \pm 0.04$ & $11.61 \pm 0.02$ & -1.07 & $14.02 \pm 0.02$ & $13.98 \pm 0.02$ & -0.17 \\
\hline 298.15 & $12.69 \pm 0.06$ & $12.61 \pm 0.03$ & -0.35 & $14.64 \pm 0.03$ & $14.87 \pm 0.01$ & 0.79 \\
\hline 300.15 & $14.27 \pm 0.04$ & $13.33 \pm 0.02$ & -3.54 & $15.31 \pm 0.05$ & $15.49 \pm 0.03$ & 0.61 \\
\hline 303.15 & $16.01 \pm 0.03$ & $14.48 \pm 0.02$ & -5.53 & $16.27 \pm 0.01$ & $16.47 \pm 0.01$ & 0.67 \\
\hline 305.15 & $16.36 \pm 0.02$ & $15.30 \pm 0.02$ & -3.74 & $16.98 \pm 0.04$ & $17.16 \pm 0.02$ & 0.60 \\
\hline 308.15 & $16.64 \pm 0.04$ & $16.61 \pm 0.03$ & -0.08 & $18.00 \pm 0.03$ & $18.25 \pm 0.02$ & 0.78 \\
\hline 310.15 & $17.50 \pm 0.02$ & $17.56 \pm 0.01$ & 0.17 & $19.01 \pm 0.03$ & $19.02 \pm 0.01$ & 0.01 \\
\hline 313.15 & $17.62 \pm 0.04$ & $19.08 \pm 0.01$ & 4.56 & $20.49 \pm 0.04$ & $20.22 \pm 0.01$ & -0.84 \\
\hline \multicolumn{4}{|c|}{ Water } & & & \\
\hline 293.15 & $0.02 \pm 0.00$ & $0.02 \pm 0.00$ & 2.57 & & & \\
\hline 295.15 & $0.03 \pm 0.00$ & $0.03 \pm 0.00$ & -0.06 & & & \\
\hline 298.15 & $0.04 \pm 0.00$ & $0.04 \pm 0.01$ & -1.68 & & & \\
\hline 300.15 & $0.05 \pm 0.01$ & $0.05 \pm 0.01$ & -1.39 & & & \\
\hline 303.15 & $0.06 \pm 0.01$ & $0.06 \pm 0.01$ & -0.34 & & & \\
\hline 305.15 & $0.08 \pm 0.01$ & $0.08 \pm 0.01$ & -0.77 & & & \\
\hline 308.15 & $0.10 \pm 0.00$ & $0.10 \pm 0.01$ & -0.44 & & & \\
\hline 310.15 & $0.12 \pm 0.01$ & $0.12 \pm 0.01$ & 0.32 & & & \\
\hline 313.15 & $0.14 \pm 0.02$ & $0.16 \pm 0.01$ & 1.97 & & & \\
\hline
\end{tabular}

$x_{c i}=$ Calculated solubility of solute (BHT). 
where $N$ is the number of experimental points and $x_{c i}$ is the solubility calculated by equation (2). The relative deviations $(R D)$ between the experimental and calculated values of solubilities are also calculated by equation (5) and are given in Table 4.

$$
\text { Relative Deviation }=\left(\frac{x-x_{c i}}{x}\right)
$$

Further, the activity coefficient $\gamma$ for BHT in different solvents were also calculated as $\mathrm{x}_{2}{ }^{\mathrm{id}} / \mathrm{x}$ and is reported in Table 6 . It is observed that activity coefficient is minimum for butanol and maximum for water. This trend is similar to solubility of BHT in the studied solvents.

Table 5. Constants A and B of equation 2, Absolute Average Deviation $(A A D)$, and root mean Square Deviation ( $r m s d$ ) of BHT in different solvents.

\begin{tabular}{|c|c|c|c|c|}
\hline Solvents & $\mathrm{A}$ & $\mathrm{B}$ & $10^{-5} \mathrm{rmsd}$ & $100 \mathrm{AAD}$ \\
\hline Methanol & -13.27 & 0.0335 & 1.2655 & 0.4297 \\
\hline Ethanol & -14.21 & 0.03873 & .5965 & 0.3738 \\
\hline Propanol & -10.30 & 0.00276 & 70.200 & -0.5413 \\
\hline Butanol & -8.02 & 0.0205 & 2.8761 & 0.2046 \\
\hline Water & -35.71 & 0.0935 & 0.0045 & 0.0194 \\
\hline
\end{tabular}

Table 6. The activity coefficient for BHT in different solvents at different temperatures.

\begin{tabular}{|c|c|c|c|c|c|}
\hline Temp. (K) & \multicolumn{5}{|c|}{ Activity coefficient } \\
\hline & Methanol & Ethanol & Propanol & Butanol & Water \\
\hline 293.15 & 11.93793 & 6.182546 & 3.332926 & 2.417476 & 1641.466 \\
\hline 295.13 & 10.89133 & 6.234895 & 2.899462 & 2.454823 & 1147.221 \\
\hline 298.15 & 9.819304 & 6.332862 & 2.909423 & 2.521898 & 923.0146 \\
\hline 300.15 & 9.644678 & 6.119487 & 2.710242 & 2.526137 & 773.5032 \\
\hline 305.15 & 9.482994 & 5.878111 & 2.588425 & 2.547061 & 690.678 \\
\hline 305.13 & 9.131989 & 5.582619 & 2.651403 & 2.55459 & 542.2118 \\
\hline 308.15 & 8.759692 & 5.257799 & 2.790046 & 2.579243 & 464.2637 \\
\hline 310.15 & 8.718079 & 4.807891 & 2.77484 & 2.555639 & 404.6642 \\
\hline 313.15 & 8.725521 & 4.741265 & 2.946473 & 2.533765 & 370.8346 \\
\hline
\end{tabular}




\section{2. Thermodynamic functions of dissolution}

From the experimental solubility data, some thermodynamic parameters such as heat of solution $\left(\Delta H_{s o l}\right)$, Gibbs energies of dissolution $\left(G_{s o l}\right)$ and enthalpy of dissolution $\left(\Delta H_{s o l}\right)$ were also evaluated. The heat of solution $\left(\Delta H_{s o l}\right)$ can be calculated using Von't Hoff equation [12]. i.e. from the slope of the plot of $\ln x$ verses $1 / T$, if the solubility is low as in case of water. However, in more recent treatments, the mean harmonic temperature, $T_{h m}$ has been introduced in Van't Hoff equation. The mean harmonic temperature, $T_{h m}$ is calculated as [13-14]

$$
T_{h m}=\frac{n}{\sum_{1}^{n}\left(\frac{1}{T}\right)}
$$

where $\mathrm{n}$ is the number of tested temperatures. In the present case, the $T_{h m}$ value obtained is $302.79 \mathrm{~K}$. Thus, the modified van't Hoff equation can be written as:

$$
\left(\frac{\partial \ln x}{\partial\left(\frac{1}{T}-\frac{1}{302.79 K}\right)}\right)=-\frac{\Delta H_{s o l}}{R}
$$

From the slopes of the plots of $\ln x$ against $\left[(1 / T)-\left(1 / T_{h m}\right)\right], \Delta H_{s o l}$ values for all the solvents were evaluated. Further, the standard Gibbs energies of the dissolution process $\left(\Delta G_{s o l}\right)$ were also calculated using following relation:

$$
\Delta G_{\text {sol }}=-R T_{h m} . \text { Intercept }
$$

Using these $\Delta H_{s o l}$ and $\Delta G_{s o l}$ values, the standard entropies of solutions $\left(\Delta S_{\text {sol }}\right)$ were obtained from the equation

$$
\Delta S_{s o l}=\left(\frac{\Delta H_{s o l}-\Delta G_{s o l}}{T_{h m}}\right)
$$

These thermodynamic values for different solvents are summarized in Table 7. As evident from Table 7, all the thermodynamic parameters are positive for the selected solvents. The enthalpy of solution is maximum in water. Among alcohols, it is maximum for methanol and minimum for butanol. The same is true for entropy and Gibb's energy of the dissolution values. The positive $\Delta G_{s o l}$ suggests that the dissolution process is not spontaneous. This is explained by the experimental solubilities (Table 4), which is much less in comparison to the ideal solubility of BHT (Table 3). The positive enthalpy suggests that the dissolution process is endothermic.

The values of thermodynamic functions calculated from ideal solubility are also reported in Table 7. In this case, Gibb's energy of dissolution is negative which is due to higher ideal solubility of BHT. However, enthalpy and entropy values are positive. In order to 
compare the relative contributions to the dissolution process from enthalpy $\% \zeta_{\mathrm{H}}$ and from entropy $\% \zeta_{\mathrm{s}}$, following equations were used:

$$
\begin{aligned}
& \% \zeta_{H}=100 \frac{\left|\Delta H_{\text {sol }}\right|}{\left|\Delta H_{\text {sol }}\right|+\left|T \Delta S_{\text {sol }}\right|} \\
& \% \zeta_{T S}=100 \frac{\left|T \Delta S_{\text {sol }}\right|}{\left|\Delta H_{\text {sol }}\right|+\left|T \Delta S_{\text {sol }}\right|}
\end{aligned}
$$

Table 7. Thermodynamic functions of experimental and ideal solubilities of BHT.

\begin{tabular}{|c|c|c|c|c|c|}
\hline Solvent & $\begin{array}{c}\Delta \mathrm{G} \\
(\mathrm{KJ} / \mathrm{mole})\end{array}$ & $\begin{array}{c}\Delta \mathrm{H} \\
(\mathrm{KJ} / \mathrm{mole})\end{array}$ & $\begin{array}{c}\Delta \mathrm{S} \\
(\mathrm{J} / \mathrm{mole})\end{array}$ & $\% \zeta_{\mathrm{H}}$ & $\% \zeta_{\mathrm{S}}$ \\
\hline Methanol & 7.9348 & 29.7176 & 68.6341 & 58.0153 & 41.9847 \\
\hline Ethanol & 6.5931 & 28.1156 & 74.3834 & 56.3847 & 43.6153 \\
\hline Propanol & 4.8586 & 21.2007 & 53.9718 & 56.4724 & 43.5285 \\
\hline Butanol & 4.5615 & 15.6054 & 36.4736 & 58.5593 & 41.4407 \\
\hline Water & 18.6866 & 72.3235 & 177.1420 & 57.4185 & 42.5815 \\
\hline $\mathrm{x}_{2}{ }^{\text {id }}$ & -1.0472 & 7.2389 & 27.3663 & 46.6273 & 53.3727 \\
\hline
\end{tabular}

The values are reported in Table 7. It is clear from these values that the main contributor to the standard Gibb's energy of the dissolution process is enthalpy for all the solvents. However, the respective contributions are reverse with respect to their ideal solubilities.

\section{3. Thermodynamic functions of mixing}

The dissolution process can be represented by the hypothetical stages:

$$
\text { Solute }_{(\text {Solid) }} \rightarrow \text { Solute }_{(\text {Liquid })} \rightarrow \text { Solute }_{(\text {Solution })}
$$

where fusion and mixing are the respective partial processes towards the drug dissolution process at $T_{h m}$. This approximation allows one to calculate the partial thermodynamic contributions to the dissolution process by the following equations:

$$
\begin{gathered}
\Delta H_{\text {sol }}=\Delta H_{\text {fus }}^{302.79}+\Delta H_{m i x} . \\
\Delta S_{\text {sol }}=\Delta S_{f u s}^{302.79}+\Delta S_{\text {mix }} .
\end{gathered}
$$

where $\Delta H_{f u s}{ }^{302.79}$ and $\Delta S_{f u s}{ }^{302.79}$ represent the thermodynamic functions of the fusion process at the harmonic temperature $(302.79 K)$. The $\Delta H_{f u s}{ }^{302.79}$ values were calculated as: 


$$
\Delta H_{f u s}^{302.79}=\Delta H_{f u s}-\Delta C_{p}\left(T_{f u s}-T_{h m}\right)
$$

$\Delta C_{p}$ is replaced by $\Delta S_{f u s}$ and value of $\Delta H_{f u s}{ }^{302.79}$ was found to be $17.4820 \mathrm{KJ} / \mathrm{mole}$ which is quite different from enthalpy value calculated for the ideal dissolution process (Table 5). Similarly, entropy of fusion $\Delta \mathrm{S}_{f u s}{ }^{302.79}$ was found to be $57.7365 \mathrm{~J} / \mathrm{mole}$ which is quite different from that calculated for the ideal dissolution process (Table 7).

So, $\Delta H_{m i x}$ values were evaluated from equation (12) using both $\Delta H_{f u s}{ }^{302.79}=17.4820$ $\mathrm{KJ} /$ mole as well as $\Delta H_{\text {sol }}$ id (given in Table 7). Similarly, $\Delta S_{\text {mix }}$ values were also evaluated from equation (13) using both $\Delta S_{\text {fus }}{ }^{302.79}=57.7365 \mathrm{~J} / \mathrm{mole}$ as well as $\Delta S_{\text {sol }}$ id (given in Table 7). All these values are given in Table 8 .

Table 8. Thermodynamic functions relative to mixing process.

\begin{tabular}{|c|c|c|c|c|c|}
\hline Solvent & Methanol & Ethanol & Propanol & Butanol & Water \\
\hline$\Delta \mathrm{H}_{\text {mix }}$ & $\begin{array}{c}11.2345 \\
(21.4787)\end{array}$ & $\begin{array}{c}11.6336 \\
(21.8767)\end{array}$ & $\begin{array}{c}3.7187 \\
(13.9618)\end{array}$ & $\begin{array}{c}-1.8766 \\
(8.3665)\end{array}$ & $\begin{array}{c}54.8415 \\
(65.0846)\end{array}$ \\
\hline$\Delta \mathrm{S}_{\text {mix }}$ & 10.8977 & 16.6469 & -3.7647 & -21.2629 & 119.4056 \\
& $(41.2678)$ & $(47.0171)$ & $(26.6055)$ & $(9.1073)$ & $(149.7757)$ \\
\hline \multirow{2}{*}{$\Delta \mathrm{G}_{\text {mix }}$} & 7.9348 & 6.5931 & 4.8586 & 4.5616 & 18.6866 \\
& $(8.9832)$ & $(7.6404)$ & $(5.9059)$ & $(5.6089)$ & $(19.7340)$ \\
\hline
\end{tabular}

*Values in parenthesis are those calculated using ideal dissolution values.

By analyzing the partial contribution by ideal solution (related to solute fusion process) and mixing processes to the enthalpy and entropy of solution, it is found that both $\Delta H_{f u s}{ }^{302.79}$ and $\Delta S_{f u s}{ }^{302.79}$ are positive. However, the contribution of the thermodynamic functions relative to the mixing process towards the solution process is varying. If values calculated from $\Delta H_{f u s}{ }^{302.79}$ and $\Delta S_{f u s}{ }^{302.79}$ are considered, $\Delta \mathrm{H}_{\text {mix }}$ value is negative for butanol where as $\Delta S_{\text {mix }}$ values are negative for both propanol and butanol and if these values given in parenthesis are compared for different solvents, again $\Delta H_{\text {mix }}$ value is minimum for butanol and $\Delta S_{\text {mix }}$ values are minimum for both propanol and butanol. However, these values are positive. Interestingly, there is not much difference in $\Delta G_{m i x}$ values evaluated by both ways. These values are positive for all the selected solvents and minimum for propanol and butanol.

The net variation in $\Delta H_{\text {mix }}$ values is due to contribution of various kinds of interactions. The enthalpy of cavity formation is endothermic because energy must be supplied to overcome the cohesive forces of the solvent which is generally hydrogen bonding as in case of water. This causes a decrease of solubility. The enthalpy of solute-solvent interaction is exothermic due to various types of interactions. The structure of solvent molecules around non polar groups of solute contributes to a decrease in the net enthalpy of mixing as observed for alcohols.

Further, the minimum solubility of water suggests that BHT structure leads to water association which is not favorable for solubility of BHT in water whereas butanol could form maximum hydrogen bonding with BHT which causes increase in solubility. Thus, it is concluded that solubility of BHT is maximum in butanol and minimum in water. The 
dissolution process of BHT in the studied solvents is endothermic in nature and is not spontaneous.

\section{CONCLUSION}

The solubility of BHT increases with increasing $\mathrm{CH}_{2}$ group in alcohols and increases with increase in dielectric constant in selected primary alcohols. Further, solubility is higher in butanol and minimum in water. Further, the minimum solubility of water suggests that BHT structure leads to water association which is not favorable for solubility of BHT in water whereas butanol could form maximum hydrogen bonding with BHT which causes increase in solubility. The dissolution process of BHT in the studied solvents is endothermic in nature and is not spontaneous.

\section{ACKNOWLEDGEMENT}

The authors are thankful to Head of Department of Chemistry, Saurashtra University, Rajkot for providing necessary facility for entire research of work.

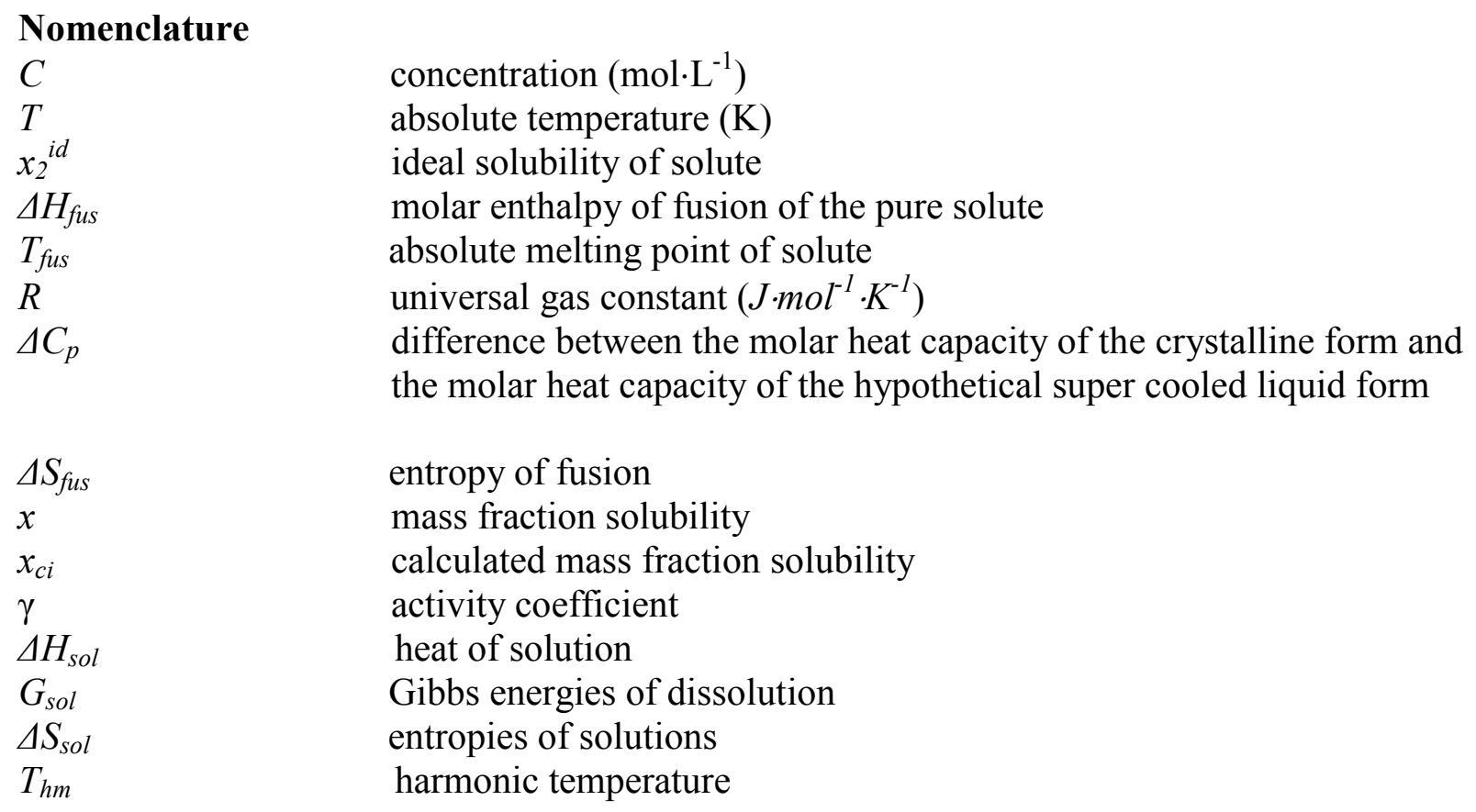

\section{Greek symbol} $\gamma$

activity coefficient

\section{Abbreviation}

BHT

AAD

rmsd

$\mathrm{RD}$
Butylated hydroxytoluene absolute average deviations root-mean-square deviations relative deviations 


\section{References}

[1] Grantham P. H., Weisburger J. H., Weisburger E. K., Food and Cosmetics Toxicology 11(2) (1973) 209-217.

[2] Burton G. W., Ingold K. U., J. Ame. Chem. Soc. 103(1) (1981) 6472-6477.

[3] Lee H. B., Hanguk Yongyang Hakhoechi, 8(2) (1975) 95-100.

[4] Moy S. A., Sweet F., Proc. Polyurethanes Conf. 94 (1994) 214-219.

[5] Fujisawa S., Kadomab Y., Yokoe I., Chem. and Phys. of Lipids. 130(2) (2004) 189195.

[6] Cottrell S., Andrews C. M., Clayton D., Powell,C. J., Food and Chemical Toxicology 32(7) (1994) 589-594.

[7] Bouftira I., Abdelly C., Sfar S., African J. Biotech. 6(9) (2007) 1136-1139.

[8] Jimenez J. A., Martinez F., J. Braz. Chem. Soc. 17 (2006) 125-134.

[9] Stott P. W., Williams A. C., Barry B. W., Int. J. Pharma. 219 (2001) 161-176.

[10] Apelblat A. Manzurola E., J. Chem. Thermodyn. 31 (1999) 85-91.

[11] Gao J., Wang Z. W., Xu D. M., Zhang R. K., J. Chem.\& Eng. Data 52 (2007) 189191.

[12] Nordstrom F. L., Rasmuson A. C., J. Chem. \& Eng. Data 51 (2006) 1668-1671.

[13] Krug R. R., Hunter W. G., Grieger R. A., J. Phys. Chem. 80 (1976) 2341-2351.

[14] Bustamante P., Romero S., Pena A., Escalera B., Reillo A., J. Pharm. Sci. 87 (1998) 1590-1596. 